\title{
Theory of the Shubnikov-de Haas effect in quasi-two-dimensional metals
}

\author{
P.D. Grigoriev \\ Grenoble High Magnetic Field Laboratory, MPI-FKF and CNRS, \\ BP 166, F-38042 Grenoble Cedex 09, France. \\ L.D.Landau Institute for Theoretical Physics, 142432 Chernogolovka, Russia.
}

(October 27, 2018)

\begin{abstract}
The Shubnikov - de Haas effect in quasi-two-dimensional normal metals is studied. The interlayer conductivity is calculated using the Kubo formula. The electron scattering on short-range is considered in the self-consistent Born approximation. The result obtained differs from that derived from the Boltzmann transport equation. This difference is shown to be a general feature of conductivity in magnetic field. A detailed description of the two new qualitative effects - the field-dependent phase shift of beats and of the slow oscillations of conductivity is provided. The results obtained are applicable to strongly anisotropic organic metals and to other quasi-two-dimensional compounds.
\end{abstract}

\section{INTRODUCTION}

Magnetic quantum oscillations were discovered long ago and were frequently used as a powerful tool of studying the geometry of Fermi surfaces and other electronic properties of various metals. In recent years, quasi-two-dimensional (quasi-2D) organic metals [2] attract great interest because many new unconventional effects are very pronounced in these compounds. These effects are high- $\mathrm{T}_{c}$ superconductivity, spin and charge density waves, strong anisotropic magnetic quantum oscillations etc. Much work was devoted to studying magnetic quantum oscillations in these compounds (for a review see e.g. [3]). The quantum oscillations of magnetization are a thermodynamic effect that is completely determined by the density-of-states distribution. Any exact calculation of the electron density of states (DoS) is a very complicated problem but a semi-phenomenological description of magnetization oscillations in quasi$2 \mathrm{D}$ compounds was recently provided in a number of theoretical papers [ [ [6]. The chemical potential oscillations and the arbitrary electron reservoir due to the open sheets of the Fermi surface create no principal difficulties [6]. Since the number of occupied LLs is very large $\left(n_{F}>100\right)$ in most quasi-2D organic metals, the effect of the electronelectron interaction is reduced (as in the Fermi liquid) and can be taken into account via the renormalization of the electron effective parameters. Differences in Landau level shape (which depends on a particular compound) lead to only limited quantitative differences in magnetization curves yielding no qualitatively new effects. On the qualitative level, therefore, the de Haas-van Alphen (dHvA) effect in quasi-2D normal metals is believed to be well understood.

Attempts of theoretical description of the quasi-2D Shubnikov-de Haas ( $\mathrm{SdH})$ effect were not as successful although some work on this subject appeared in recent years [5.7] 10. There are still many open qualitative questions.

One of these open questions is the origin of the phase shift in the beats of the resistivity oscillations with respect to those in the magnetization. The beat behavior of the oscillations in quasi-2D metals is known reliably to originate from a slight warping of their Fermi surfaces in the direction normal to the 2D plane. The superposition of the contributions from the maximum and minimum cyclotron orbits leads to an amplitude modulation of the $k$-th harmonic by the factor $\cos (2 \pi k \Delta F / 2 B-\pi / 4)$, where $B$ is the magnetic field and $\Delta F=(c \hbar / 2 \pi e)\left(A_{\max }-A_{\min }\right)$ is the difference between the oscillation frequencies caused by the extreme orbits with the $k$-space areas $A_{\max }$ and $A_{\min }$, respectively [1]. From the beat frequency one can readily evaluate the warping of the Fermi surface and hence the interlayer transfer integral $4 t \approx \epsilon_{F} \Delta F / F$ (see e.g. 12,11]). The situation becomes less clear when the warping is so weak that less than one half of the beat period can be observed experimentally. In principle, an observation of one single node would already be quite informative [14], provided the phase offset (i.e. the phase of the beat at $1 / B \rightarrow 0$ ) is known. In the standard Lifshitz-Kosevich (L-K) theory 13 this phase offset is strictly determined by geometrical reasons and is equal to $-\pi / 4$ for both the $\mathrm{dHvA}$ and $\mathrm{SdH}$ effects [1]. However recent experiments on layered organic metals $\kappa-(\mathrm{BEDT}-\mathrm{TTF})_{2} \mathrm{Cu}\left[\mathrm{N}(\mathrm{CN})_{2}\right] \mathrm{Br}$ [14] and $(\mathrm{BEDT}-\mathrm{TTF})_{4}\left[\mathrm{Ni}(\mathrm{dto})_{2}\right]$ [15] revealed a significant difference in the node positions of the beats of $\mathrm{dHvA}$ and $\mathrm{SdH}$ signals. The phase shift in the latter compound was estimated to be as big as $\pi / 2$.

Another very interesting phenomenon (also not explained in the framework of the standard theory) is slow oscillations of magnetoresistance that were observed in a number of quasi-2D organic metals [3, 16 [19]. The behavior of slow oscillations resembles that of the $\mathrm{SdH}$ effect that lead to a suggestion of additional, very small Fermi surface pockets in these materials. However, band structure calculations (basically giving a good description of the electron band structure and of the Fermi surface topology in organic metals) show no evidence of such small pockets in any of these compounds. Moreover, while slow oscillations are often very pronounced in magnetoresistance, sometimes even dominating the oscillation spectrum, thus far no analogous observation in oscillating magnetization (dHvA effect) was 
reported. Certainly, slow oscillations carry useful information about the compounds but one needs some theoretical explanation and, desirably, a quantitative description of the phenomenon to extract this information. Note that in both cases the oscillation spectrum was strongly dominated by the first harmonic when no substantial deviations from the standard L-K theory are expected.

An explanation and the qualitative description of phase shift of beats (which is based on the Boltzmann transport equation) was proposed recently [20] together with a comparative experimental study of this effect. An idea that the slow oscillation may arise as an entanglement of different rapidly oscillating contributing factors in conductivity which have slowly oscillating amplitudes due to beats was also recently suggested in [21] and proved by presenting experimental results on temperature and angular dependences of slow oscillations. In this paper we give a more accurate theoretical description of these phenomena as well as a detailed calculation of the interlayer conductivity in quasi-2D metals in strong magnetic field.

In sec. II the general formula (10) is derived. The calculation is performed starting from the Kubo formula. The result of this calculation differs from that obtained using the Boltzmann transport equation. The origin of this difference is pointed out. The additional term is shown to be a general feature of conductivity in magnetic field. However it becomes essential only in the quasi-2D case. The simple explicit formula for interlayer conductivity is obtained in the self-consistent Born approximation in sec. III. A discussion of the reliability and possible application of the results obtained is given in sec. IV.

\section{GENERAL FORMULA FOR INTERLAYER CONDUCTIVITY}

We consider a quasi-2D metal in magnetic field perpendicular to the conducting layers: $\vec{B} \| \vec{z}$. The electron spectrum of quasi-2D electron gas in magnetic field is then given by

$$
\epsilon\left(n, k_{z}\right)=\hbar \omega_{c}(n+1 / 2)-2 t \cos \left(k_{z} d\right)
$$

where $t$ is the interlayer transfer integral, $k_{z}$ is the wavevector perpendicular to the layers, $d$ is the interlayer distance, $\omega_{c}=e B / m^{*} c$ is the cyclotron frequency. Both $\hbar \omega_{c}$ and $t$ are assumed to be much smaller than the Fermi energy.

To calculate conductivity we use the Kubo formula 22]. The procedure is similar to that in three-dimensional metals without magnetic field ( 22], $\S 7.1 .2$ ). In magnetic field only the new set of quantum numbers $m \equiv\left\{n, k_{z}, k_{y}\right\}$ should be used instead of momentum $\vec{p}$ and the alternative dispersion relation (11). The evaluation of the Kubo formula without vertex corrections gives

$$
\sigma_{z z}=\frac{e^{2} \hbar}{V} \sum_{m} v_{z}^{2}(m) \int \frac{d \epsilon}{2 \pi} A^{2}(m, \epsilon)\left(-n_{F}^{\prime}(\epsilon)\right)
$$

where the volume $V$ normalizes the sum over quantum numbers $m, e$ is the electron charge, the limits of the integral over $\epsilon$ are $(-\infty ; \infty), n_{F}^{\prime}(\epsilon)$ is the derivative of the Fermi distribution function:

$$
-n_{F}^{\prime}(\epsilon)=1 /\left\{4 T \cosh ^{2}[(\epsilon-\mu) / 2 T]\right\}
$$

and $A(m, \epsilon)$ is the spectral function that is related to the electron Green's function $G^{R}(m, \epsilon)$ or to the retarded self-energy part $\Sigma^{R}(m, \epsilon)$ :

$$
A(m, \epsilon) \equiv-2 \operatorname{Im} G^{R}(m, \epsilon)=\frac{-2 \operatorname{Im} \Sigma^{R}(m, \epsilon)}{\left[\epsilon-\epsilon(m)-\operatorname{Re} \Sigma^{R}(m, \epsilon)\right]^{2}+\left[\operatorname{Im} \Sigma^{R}(m, \epsilon)\right]^{2}}
$$

Formula (2) is close to the corresponding formula without magnetic field [ [22], formula (7.1.10)] until the self-energy part $\Sigma^{R}(m, \epsilon)$ is specified. It arises mainly from impurity scattering. The main contribution to resistivity comes from the short-range impurity scattering. We approximate short-range impurities by point-like ones. Then, if one does not take into account the diagrams with intersections of impurity lines in the self-energy (the contribution of such diagrams at finite $k_{z}$ dispersion of electrons is usually small) the electron self-energy depends only on electron energy and not on electron quantum numbers. This fact greatly simplifies the calculations because the sum over quantum numbers $m$ in formula (2) can be now computed analytically. The constant part of the real part $\operatorname{Re} \Sigma^{R}(\epsilon)$ of electron self-energy produces only a constant shift of the chemical potential. It does not influence the physical effects and, hence, is omitted in the subsequent calculations. The small oscillating part $\operatorname{Re} \tilde{\Sigma}^{R}(\epsilon)$ of $\operatorname{Re} \Sigma^{R}(\epsilon)$ enters the final expression for conductivity in the second order in small damping factors. Hence, it can affect slow oscillations of conductivity and should be kept in accurate quantitative analysis. It always come in the combination $\epsilon^{*} \equiv \epsilon-\operatorname{Re} \tilde{\Sigma}^{R}(\epsilon)$. 
On the contrary, the imaginary part of self-energy $\operatorname{Im} \Sigma^{R}(\epsilon)$ is very important since it describes the momentum relaxation of electrons.

Performing the summation over $k_{y}$ in (2) and changing integration over $k_{z}$ by integration over energy $\epsilon\left(n, k_{z}\right)$ we get

$$
\begin{aligned}
\sigma_{z z} & =e^{2} \hbar N_{L L} \sum_{n} \int_{0}^{\pi} \frac{d\left(k_{z} d\right)}{\pi} v_{z}^{2}\left(k_{z}\right) \int \frac{d \epsilon}{2 \pi} A^{2}\left(\epsilon\left(k_{z}, n\right), \epsilon\right)\left(-n_{F}^{\prime}(\epsilon)\right)= \\
& =e^{2} N_{L L} d \int \frac{d \epsilon^{\prime}}{\pi} \sum_{n}\left|v_{z}\left(\epsilon^{\prime}, n\right)\right| \int \frac{d \epsilon}{2 \pi} A^{2}\left(\epsilon^{\prime}, \epsilon\right)\left(-n_{F}^{\prime}(\epsilon)\right)
\end{aligned}
$$

where $N_{L L} \equiv B / \Phi_{0} d$ is the electron density on one Landau level and the electron velocity $v_{z}(\epsilon, n)$ is given by (A2). To go further we have to transform the sum over LLs to a sum over harmonics. This can be done using the Poisson summation formula (Appendix A). Substituting (A3) into (5) we obtain:

$$
\begin{aligned}
\sigma_{z z} & =e^{2} N_{L L} \int \frac{d \epsilon^{\prime}}{2 \pi} \sum_{k=-\infty}^{\infty}(-1)^{k} \frac{2 t d^{2}}{\hbar k} \exp \left(\frac{2 \pi i k \epsilon^{\prime}}{\hbar \omega_{c}}\right) J_{1}\left(\frac{4 \pi k t}{\hbar \omega_{c}}\right) \int \frac{d \epsilon}{2 \pi} A^{2}\left(\epsilon^{\prime}, \epsilon\right)\left(-n_{F}^{\prime}(\epsilon)\right)= \\
& =e^{2} N_{L L} \sum_{k=-\infty}^{\infty}(-1)^{k} \frac{2 t d^{2}}{\hbar k} J_{1}\left(\frac{4 \pi k t}{\hbar \omega_{c}}\right) \int \frac{d \epsilon}{2 \pi}\left(-n_{F}^{\prime}(\epsilon)\right) I_{z}(\epsilon, k)
\end{aligned}
$$

where one should use the expansion $J_{1}(k x) / k=x / 2$ for the zeroth harmonic $k=0$, and the integral $I_{z}(\epsilon, k)$ over $\epsilon^{\prime}$ can be easily evaluated with the spectral function (舟):

$$
\begin{aligned}
I_{z}(\epsilon, k) & \equiv \int \frac{d \epsilon^{\prime}}{2 \pi} A^{2}\left(\epsilon^{\prime}, \epsilon\right) \exp \left(\frac{2 \pi i k \epsilon^{\prime}}{\hbar \omega_{c}}\right)= \\
& =\int \frac{d \epsilon^{\prime}}{2 \pi}\left(\frac{-2 \operatorname{Im} \Sigma^{R}(\epsilon)}{\left[\epsilon^{*}-\epsilon^{\prime}\right]^{2}+\left[\operatorname{Im} \Sigma^{R}(\epsilon)\right]^{2}}\right)^{2} \exp \left(\frac{2 \pi i k \epsilon^{\prime}}{\hbar \omega_{c}}\right)= \\
& =\exp \left(\frac{2 \pi i k \epsilon^{*}}{\hbar \omega_{c}}\right)\left(\frac{1}{\left|\operatorname{Im} \Sigma^{R}(\epsilon)\right|}+\frac{2 \pi k}{\hbar \omega_{c}}\right) R_{D}(k, \epsilon)
\end{aligned}
$$

where $\epsilon^{*} \equiv \epsilon-\operatorname{Re} \tilde{\Sigma}^{R}(\epsilon)$ and

$$
R_{D}(k, \epsilon)=\exp \left(-2 \pi|k|\left|\operatorname{Im} \Sigma^{R}(\epsilon)\right| / \hbar \omega_{c}\right)
$$

has the form similar to that of the usual Dingle factor $R_{D}(k)=\exp \left(-2 \pi^{2} k k_{B} T_{D} / \hbar \omega_{c}\right)$. Collecting formulas $(6)$ and (8) we get

$$
\begin{aligned}
\sigma_{z z}= & e^{2} N_{L L} \int \frac{d \epsilon}{2 \pi}\left(-n_{F}^{\prime}(\epsilon)\right) \sum_{k=-\infty}^{\infty} \frac{(-1)^{k} 2 t d^{2}}{\hbar k} J_{1}\left(\frac{4 \pi k t}{\hbar \omega_{c}}\right) \times \\
& \times \exp \left(\frac{2 \pi i k \epsilon^{*}}{\hbar \omega_{c}}\right)\left(\frac{1}{\left|\operatorname{Im} \Sigma^{R}(\epsilon)\right|}+\frac{2 \pi k}{\hbar \omega_{c}}\right) R_{D}(k, \epsilon) .
\end{aligned}
$$

Note that this expression has additional term $2 \pi k / \hbar \omega_{c}$ near the standard $1 /\left|\operatorname{Im} \Sigma^{R}(\epsilon)\right|$ term in round brackets in the second line. This term can not be obtained from the Boltzmann transport equation (compare, for example, with the results of [20] and [21]) and it arises only due to quantization of electron energy spectrum in magnetic field. This quantization results in fast oscillations of the mean square electron velocity as function of energy which give rise to the rapidly oscillating factor $\exp \left(2 \pi i k \epsilon^{\prime} / \hbar \omega_{c}\right)$ in $($ 7). This factor appears only in magnetic field. The derivative of this factor with respect to $\epsilon^{\prime}$ comes after the integration over $\epsilon^{\prime}$ in $(\sqrt{7})$ because the function $A^{2}\left(\epsilon^{\prime}, \epsilon\right)=\left(G^{A}-G^{R}\right)^{2}$ has one second-order pole in each complex half-plane. These second-order poles arise from the combinations $\left(G^{A}\right)^{2}$ and $\left(G^{R}\right)^{2}$, where $G^{A}$ and $G^{R}$ are the advanced and retarded Green's functions respectively. So, the additional term in (10) has quantum origin; it appears due to fast oscillations of the mean squared velocity at the Fermi level in magnetic field.

To go farther, we need an explicit form of the electron self-energy which enters formula (10). It is calculated is in the next section. 


\section{CONDUCTIVITY IN SELF-CONSISTENT BORN APPROXIMATION}

We consider electron scattering only by short-range impurities because these impurities make the main contribution to the relaxation of electron momentum. To calculate the electron self-energy we use the self-consistent Born approximation. The graphical representation of the Dyson equation for the irreducible self-energy part in self-consistent Born approximation is shown in fig. 1. By such approximation we neglect multiple scattering on one impurity (no more than two dash lines go to one impurity in fig. 1). The single dash line in fig. 1 corresponds to the first-order term which leads only to a constant shift of the chemical potential and, hence, can be omitted.

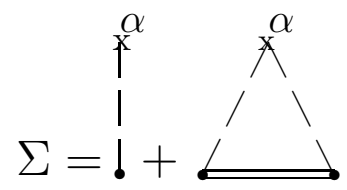

FIG. 1. The Dyson equation for the irreducible self-energy in self-consistent Born approximation. The double solid line symbolizes exact electron Green's function.

The corresponding analytical expression is

$$
\Sigma^{R}(m, \epsilon)=\left\langle\sum_{i} U^{2} G\left(r_{i}, r_{i}, E\right)\right\rangle=C_{i} U^{2} \int d^{3} r G(r, r, E)
$$

where $\sum_{i}$ is a sum over all impurities and the brackets $\langle.$.$\rangle denote averaging over impurity positions, C_{i}$ is concentration of impurities which are assumed to be uniformly distributed [24]. The electron Green's function $G(r, r, E)$ in formula (11) contains the self-energy determined by the same formula (11) (this is why the approximation (11) is called self-consistent Born approximation). The Green's function is isotropic along the conducting planes. Hence, one can write

$$
G(r, r, E)=|\phi(z)|^{2} G(E),
$$

where the electron wave function $\phi(z)$ along $z$-axis does not enter the final result because it disappears after integration over $z$ in (11), and

$$
G(E)=\frac{-N_{L L}}{\hbar \omega_{c}}\left\{A(E)+i \pi\left[1+2 \sum_{k=1}^{\infty}(-1)^{k} J_{0}\left(\frac{4 \pi k t}{\hbar \omega_{c}}\right) \exp \left(2 \pi i k \frac{E-\Sigma(E)}{\hbar \omega_{c}}\right)\right]\right\} .
$$

$A(E)$ is a slowly varying function of energy which can be taken at the Fermi energy. The exact form of this function is not important for conductivity in Born approximation. Formula (13) can be derived performing the summation over electron quantum numbers $m \equiv\left\{n, k_{z}, k_{x}\right\}$ in the definition of the Green's function:

$$
G(r, r, E)=\sum_{n, k_{z}, k_{x}} \frac{\Psi_{n, k_{z}, k_{x}}^{*}(r) \Psi_{n, k_{z}, k_{x}}(r)}{E-\epsilon_{n, k_{z}}-\Sigma(E)}
$$

where $E_{n, k_{z}, k_{x}}$ is the electron energy in the state with quantum numbers $m \equiv\left\{n, k_{z}, k_{x}\right\}$ given by (11). The electron wave function $\Psi_{n, k_{z}, k_{x}}(r)$ in Landau gauge is approximately given by

$$
\Psi_{n, k_{z}, k_{x}}(r)=\frac{e^{i\left(k_{x} x+k_{z} z\right)}}{\sqrt{L_{x} L_{z}}} \chi_{n}\left(y-y_{0}\right) \phi(z)
$$

where $y_{0}=-c \hbar k_{x} / e B$ and the normalization condition $\int_{-\infty}^{\infty}\left|\chi_{n}(y)\right|^{2} d y=1$ is used to perform integration over $k_{x}$. The further calculation of the sum in $(14)$ is similar to that in (2).

The Born approximation (formula (11)) takes into account only the first term of expansion in the small parameter $\pi U N_{L L} / \hbar \omega_{c}=\pi f / d$, where $N_{L L} / \hbar \omega_{c}$ is equal to the electron density of states at the Fermi level in unit volume, $f$ is the scattering amplitude (which is constant at small wave vector $q \ll 1 / r_{0}, r_{0}$ is the range of the impurity potential). For short-range impurities the parameter $f / d$ is usually small.

From (11) one can easily see that in Born approximation the imaginary part of self-energy is proportional to the density of states [25]: 


$$
-\operatorname{Im} \Sigma^{R}(\epsilon)=-C_{i} U^{2} \operatorname{Im} G(\epsilon)=\pi C_{i} U^{2} \times \rho(\epsilon) .
$$

The unknown coefficient $C=\pi C_{i} U^{2}$ in (15) is simply related to the average Dingle temperature $T_{D}:\left\langle\left|\operatorname{Im} \Sigma^{R}(m, \epsilon)\right|\right\rangle=$ $C \cdot\langle\rho(\epsilon)\rangle=C \cdot\left(N_{L L} / \hbar \omega_{c}\right)\left(1+n_{R}\right)=\pi k_{B} T_{D}$, where the triangular brackets mean an average value of a quantity inside, $k_{B}=1.38 \cdot 10^{-16} \mathrm{erg} / \mathrm{K}$ is the Boltzmann constant and $n_{R}$ is the density of reservoir states that exist in many organic metals due to the open sheets of the FS.

In the extreme 2D case $\left(\hbar \omega_{c} \gg t\right)$, substantial deviations from formula (15) are possible because the strong degeneracy of the LLs makes the Born approximation not applicable. Since we consider the case $2 t>\hbar \omega_{c}$ (when the beats of the oscillations exist) and $f / d \ll 1$, we shall use (11) for our subsequent calculations that are now straightforward.

From the formulas (11, 12 and 13) we have

$$
\left|\operatorname{Im} \Sigma^{R}(m, \epsilon)\right|=\pi k_{B} T_{D}\left(1+2 \sum_{k=1}^{\infty}(-1)^{k} J_{0}\left(\frac{4 \pi k t}{\hbar \omega_{c}}\right) \cos \left(\frac{2 \pi k \epsilon^{*}}{\hbar \omega_{c}}\right) R_{D}(k, \epsilon)\right) .
$$

Together with (9) this gives a nonlinear equation for $\operatorname{Im} \Sigma^{R}(m, \epsilon)$. We can solve it in the strong harmonic damping limit using iteration procedure i.e. by making an expansion in the small oscillating part which is an expansion in a parameter $\left(R_{D} \sqrt{\hbar \omega_{c} / 2 \pi^{2} t}\right)$. To treat the slow oscillation accurately one has also to pick up all second-order slowly oscillating terms. We get

$$
\left|\operatorname{Im} \Sigma^{R}(\epsilon)\right| \approx \pi k_{B} T_{D}\left\{1-2 J_{0}\left(\frac{4 \pi t}{\hbar \omega_{c}}\right) \cos \left(\frac{2 \pi \epsilon}{\hbar \omega_{c}}\right) R_{0 D}\right\} .
$$

where $R_{0 D}=\exp \left(-2 \pi^{2} k_{B} T_{D} / \hbar \omega_{c}\right)$. There is no slowly oscillating second-order term in the self energy in Born approximation. At this point the real part of the electron self energy was important because it canceled the contribution from the entanglement with the oscillations of the Dingle factor (9). In the second order in damping factors any combination of the form

$$
\cos \left(\frac{2 \pi\left(\epsilon-\operatorname{Re} \tilde{\Sigma}^{R}(\epsilon)\right)}{\hbar \omega_{c}}\right) \exp \left(\frac{-2 \pi\left|\operatorname{Im} \Sigma^{R}(\epsilon)\right|}{\hbar \omega_{c}}\right)=\cos \left(\frac{2 \pi \epsilon}{\hbar \omega_{c}}\right) R_{0 D}
$$

does not produce slowly oscillating term. This statement can be easily checked by substituting (11) with (13) into (16). If we neglected $\operatorname{Re} \Sigma^{R}(\epsilon)$ in (16) we would get an additional slowly oscillating term

$$
\left\{-\frac{4 \pi^{2} k_{B} T_{D}}{\hbar \omega_{c}} J_{0}^{2}\left(\frac{4 \pi t}{\hbar \omega_{c}}\right) R_{0 D}^{2}\right\}
$$

in curly brackets of (17) which arise from the mixing with the oscillating Dingle factor and enters not only the imaginary part of the self-energy but also the density of electron states (see (17)). The slow oscillations of $\rho(\epsilon)$ would result in huge slow oscillations of magnetization which are increased by an additional factor $\epsilon_{F} / \hbar \omega_{c}$. Such huge slow oscillations of magnetization have surely not been observed and the result (17) is correct. Beyond the Born approximation the relation (15) is no more valid and $\operatorname{Im} \Sigma^{R}(\epsilon)$ acquires some slow oscillating term although $\rho(\epsilon)$ does not. In the previous (unpublished) work [26] the real part of electron self-energy was disregarded and the entanglement with the oscillations of Dingle temperature (producing additional large contribution to the slow oscillations) was incorrectly taken into account.

Substituting (17) into (10) we obtain the following expression for the conductivity:

$$
\begin{aligned}
& \sigma_{z z}=e^{2} N_{L L} \int d \epsilon\left(-n_{F}^{\prime}(\epsilon)\right) \frac{2 t^{2} d^{2}}{\hbar^{2} \omega_{c} \pi k_{B} T_{D}} \times \\
& \times\left\{\frac{1-\frac{\hbar \omega_{c}}{\pi t} J_{1}\left(\frac{4 \pi t}{\hbar \omega_{c}}\right) \cos \left(\frac{2 \pi \epsilon^{*}}{\hbar \omega_{c}}\right) R_{D}(\epsilon)}{\left[1-2 J_{0}\left(\frac{4 \pi t}{\hbar \omega_{c}}\right) \cos \left(\frac{2 \pi \epsilon^{*}}{\hbar \omega_{c}}\right) R_{D}(\epsilon)\right]}-\right. \\
& \left.-\frac{2 \pi k_{B} T_{D}}{t} J_{1}\left(\frac{4 \pi t}{\hbar \omega_{c}}\right) \cos \left(\frac{2 \pi \epsilon^{*}}{\hbar \omega_{c}}\right) R_{D}(\epsilon)\right\} .
\end{aligned}
$$


If the transfer integral is large enough, $4 \pi t>\hbar \omega_{c}$, one can use the expansions of the Bessel function at large value of argument:

$$
\begin{aligned}
& J_{0}(x) \approx \sqrt{2 / \pi x} \cos (x-\pi / 4), x \gg 1 \\
& J_{1}(x) \approx \sqrt{2 / \pi x} \sin (x-\pi / 4), x \gg 1 .
\end{aligned}
$$

Then performing again expansion in small parameter $\left(R_{D} \sqrt{\hbar \omega_{c} / 2 \pi^{2} t}\right)$ and making use of the standard trigonometric formulas we get

$$
\begin{aligned}
& \sigma_{z z}=\frac{e^{2} N_{L L} 2 t^{2} d^{2}}{\hbar^{2} \omega_{c} \pi k_{B} T_{D}}\left\{1+2 \sqrt{\frac{\hbar \omega_{c}\left(1+a^{2}\right)}{2 \pi^{2} t}} \cos \left(\frac{2 \pi \mu}{\hbar \omega_{c}}\right) \times\right. \\
& \times \cos \left(\frac{4 \pi t}{\hbar \omega_{c}}-\frac{\pi}{4}+\phi_{b}\right) R_{D} R_{T}+ \\
& \left.+\frac{\hbar \omega_{c}}{2 \pi^{2} t} R_{D^{*}}^{2} \sqrt{1+a_{S}^{2}} \cos \left[2\left(\frac{4 \pi t}{\hbar \omega_{c}}-\frac{\pi}{4}+\phi_{S}\right)\right]\right\}
\end{aligned}
$$

where the phase shift of beats is

$$
\phi_{b}=\arctan (a) ; a=\frac{\hbar \omega_{c}}{2 \pi t}\left(1+\frac{2 \pi^{2} k_{B} T_{D}}{\hbar \omega_{c}}\right)
$$

and the phase of slow oscillations is

$$
\phi_{S}=\arctan \left(a_{S}\right) / 2 \quad \text { where } \quad a_{S}=\hbar \omega_{c} / 2 \pi t .
$$

The star index in the Dingle factor $R_{D^{*}}$ of slow oscillations is explained in the next section. The temperature smearing factor is given by the usual L-K expression:

$$
R_{T}=\frac{2 \pi^{2} k_{B} T / \hbar \omega_{c}}{\sinh \left(2 \pi^{2} k_{B} T / \hbar \omega_{c}\right)}
$$

It appear in the fast Shubnikov oscillations after integration over energy of a rapidly oscillating function of energy with the Fermi distribution function. The slowly oscillating term depends only on the transfer integral $t$ and is independent of energy. Hence, it does not acquire any temperature smearing.

The phase shift (20) obtained from the Kubo formula is larger than that of [ [20], formula (9)] obtained using the Boltzmann transport equation by a factor $\left(1+2 \pi^{2} k_{B} T_{D} / \hbar \omega_{c}\right)$. This difference comes from the additional term $2 \pi k / \hbar \omega_{c}$ near $1 /\left|\operatorname{Im} \Sigma^{R}(\epsilon)\right|$ in round brackets in the second line of (10). As has been noted after formula (10), this term has quantum origin. However, the result (19) and (21) concerning the slow oscillation does not differ from [ [21], formula (4)]. Thus the Boltzmann transport equation and the Kubo formula in self-consistent Born approximation give the same amplitude and phase of slow oscillations.

\section{DISCUSSION OF THE RESULTS}

In this paper a detailed calculation of the interlayer magnetotransport in quasi-2D normal metals is performed. The specific features of quasi-two-dimensionality and strong magnetic field result in pronounced qualitative effects such as the phase shift of beats of conductivity oscillations and the slow oscillations which cannot be described in the framework of the standard three-dimensional theory usually applied to quasi-2D compounds. The beats of magnetoresistance oscillations in layered compounds are used for estimating the interlayer transfer integral which strongly influences different electronic properties of strongly anisotropic compounds. The field-dependent phase shift of beats may lead to the errors in this estimate. Hence, a detailed quantitative description of this phenomenon is important.

The result of the calculation using the Kubo formula is different from that obtained using Boltzmann transport equation. An additional term in conductivity (see formula (10) and the discussion after this formula) is general for conductivity in magnetic field. It arises due to Landau quantization of electron energy spectrum. However, this term is proportional to the oscillations of the electron mean square velocity which are smaller than the oscillations of the 
electron relaxation time (or of $-\operatorname{Im} \Sigma^{R}(\epsilon) \sim \rho(\epsilon)$ ) by the same factor $\hbar \omega_{c} / 2 \pi t$ as the phase shift of beats is. Therefore, this additional term in $3 \mathrm{D}$ case is smaller than the main oscillating term by the factor of $\hbar \omega_{c} / \epsilon_{F}$.

The slow oscillations of magnetoresistance can give useful information about compounds under study. A significant feature of the slow oscillations is that their Dingle factor $R_{D *}$ is different from the factor $R_{D}$ of the Shubnikov oscillations. The usual Dingle factor includes all temperature-independent mechanisms of smearing of fast quantum oscillations. These are not only microscopical scattering events of electrons but also macroscopic spatial inhomogeneities of the sample. These inhomogeneities lead to macroscopic spatial variations of the electron energy $\epsilon^{*}$ in formula (10) which is equivalent to a local shift of the chemical potential. The total signal is an average over the entire sample and such macroscopic inhomogeneities lead to the damping of magnetic quantum oscillations similar to that caused by temperature. Since the slow oscillations do not depend on $\mu$, they are not affected by this type of smearing and the corresponding Dingle temperature $T_{D}^{*}$ of slow oscillations is determined by only short-range scatterers. One can therefore estimate relative contributions from macroscopic inhomogeneities and from local defects to the scattering rate by comparing $T_{D}$ and $T_{D}^{*}$. This role could be quite essential in organic metals. For example, such a comparison for a sample of $\beta$-(BEDT-TTF $)_{2} \mathrm{IBr}_{2}$ gives [21] $T_{D}=(0.8 \pm 0.02) \mathrm{K}$ while $T_{D}^{*}=(0.15 \pm 0.02) \mathrm{K}$, that means that the long-range crystal imperfections are important for damping of fast quantum oscillations. The relaxation of electron momentum affecting transport quantities comes, however, mainly from short-range impurities.

The slow oscillations in formula (19) do not have temperature damping factor. Hence, although the amplitude of the slow oscillations contains the square of the Dingle factor (they are a second-order effect), it can be larger than the amplitude of the fast $\mathrm{SdH}$ oscillations at $T \gtrsim T_{D}$. However, our experience disagrees with the statement that the slow oscillations do not manifest any temperature dependence (and, hence, could be seen at room temperature). Actually they do have some temperature damping because the oscillating DoS itself has some temperature dependence. The temperature damping of the DoS oscillations comes from the electron-phonon and electron-electron interactions. In normal 3D metals 13 the electron-electron (e-e) scattering rate $1 / \tau_{e e} \sim\left(k_{B} T\right)^{2} / \hbar \mu$ while the electron-phonon scattering rate $1 / \tau_{p h} \sim\left(k_{B} T / \hbar\right)\left(k_{B} T / \hbar \omega_{D}\right)^{2}$. One can estimate the effect of these scattering processes on the DoS oscillations by introducing the additional damping factor

$$
R_{T D} \approx \exp \left[-\pi\left(1 / \omega_{c} \tau_{e e}+1 / \omega_{c} \tau_{p h}\right)\right]
$$

analogous to the usual Dingle factor. This factor enters squared in the amplitude of slow oscillations. The temperature $T_{S O}$ at which the slow oscillations become damped by this factor is much higher than the characteristic temperature of the damping of fast quantum oscillations. It is approximately given by $\pi\left(1 / \omega_{c} \tau_{e e}\left(T_{S O}\right)+1 / \omega_{c} \tau_{p h}\left(T_{S O}\right)\right) \approx 1$. The above analysis of the temperature dependence of slow oscillations is very approximate. A rigorous calculation must be based on the exact calculation of the electron self-energy due to these two types of interactions. Nevertheless, the above arguments can give qualitative estimates. A more accurate calculation would be useful since the temperature dependence of slow oscillations at high enough temperature may give additional information about the electron-phonon and electron-electron interactions in various compounds where slow oscillations exist. This is important since these interactions in layered organic metals determine the superconducting and the density-wave transitions.

The entanglement with the oscillations of chemical potential contributes an additional temperature-dependent term to the slow oscillations of conductivity. This term can be easily obtained by substituting (B2) into (19). However, this term has additional damping factors $R_{T}^{2}$ and $\left(R_{D} / R_{D^{*}}\right)^{2}$ compared to the main slowly oscillating term. Therefore, this correction is as small as the second harmonic of Shubnikov oscillations is, and we can neglect it.

The slow oscillations does not appear in magnetization because there is no suitable entanglement of different oscillating quantities in magnetization. The magnetization being a thermodynamic quantity is completely determined by the electron density of states. However, the density of states does not have slowly oscillating terms. The mixing with the oscillations of the chemical potential, or with those of the Dingle factor and of $\operatorname{Re} \Sigma^{R}(\epsilon)$ does not also lead to slow oscillations of magnetization (see Appendix B).

Now we shall discuss the approximations made during the derivation of formula (19).

The first limitation of the proposed analysis is that the magnetic field is taken to be perpendicular to the conducting layers. A finite tilt angle $\theta$ of the magnetic field with respect to the normal to the conducting planes may be approximately taken into account by rescaling the Landau level separation, $\omega_{c} \rightarrow \omega_{c} \cos \theta$, and of the warping of the Fermi surface [28], $t(\theta)=t(0) J_{0}\left(k_{F} d \tan \theta\right)$, where $k_{F}$ is the in-plane Fermi momentum. But this is only a semiclassical approximation based on the assumption that the FS remains the same. Actually, the tilting of the magnetic field changes the dispersion relation and a more profound study of the effect of tilting of magnetic field on transport properties is required. The quantum mechanical calculation of the dispersion relation in tilted magnetic field in the first order of the transfer integral gives [29] $t(\theta) / t(0)=\exp \left(-g^{2} / 4\right) L_{n}^{0}\left(g^{2} / 2\right)$, where $g \equiv d \tan \theta / a_{H}$, $a_{H}=\sqrt{\hbar c / e B_{z}}$ is the magnetic length and $L_{n}^{0}(x)$ is Laguerre polynomial. This result is also approximate, but it should work satisfactory at not too great tilt angles. In the limit $n \rightarrow \infty$ the two above results coincide. 
More essential errors may come from the so-called incoherent or weakly incoherent electron interlayer transport. This means, that when the interlayer transfer integral becomes comparable to the Dingle temperature, the electron interlayer jumps an electron scattering on impurities should not be considered separately. Their entanglement may result even in qualitatively new phenomena in the quasi-2D magnetotransport [8]. In the present work we consider only the case $t>T_{D}$.

Other errors may come from the approximate expression for self-energy (15). The self-consistent Born approximation at finite $k_{z}$ dispersion works quite well, but other scattering mechanisms (especially for the calculation of the DoS) should be taken into account. An accurate study of this problem may depend on a particular type of the compound in hand. It may lead to some quantitative modifications of formula (19).

The above analysis does not take into account the vertex corrections. In our case (of point-like impurity scattering) this is right because, according to the Ward identity, the vertex $\vec{\Gamma}(m, E)=\vec{p}+m \vec{\nabla}_{p} \Sigma^{R}(m, E)$. Hence, if the retarded self-energy depends only on energy, the vertex corrections are zero. The fact that $\Sigma^{R}(m, \epsilon)$ is approximately a function of energy $\epsilon$ only is a consequence of the short-range (or point-like) impurity potential. More precisely, if one takes a point-like impurity potential and neglects all diagrams with the intersections of the impurity lines in the self-energy, then after averaging over randomly and uniformly distributed impurity positions one obtains $\Sigma^{R}(m, \epsilon)=\Sigma^{R}(\epsilon)$. The neglected graphs with the intersections of the impurity lines describe the coherent scattering on two impurities simultaneously. The contribution of such scattering is small at large enough interlayer transfer integral. In the three-dimensional case without magnetic field the vertex corrections produce an additional factor $(1-\cos \alpha)$ in the integrand for the transport scattering relaxation time where $\alpha$ is the scattering angle. But the scattering probability is independent of the scattering angle in the case of point-like impurities and the additive $\cos \alpha$ vanishes after the integration over angles. Hence, the vertex corrections vanish.

In derivation of formula (19) only first- and second-order terms in the small damping factors $R_{T}$ and $R_{D}$ were taken into account, assuming the harmonic damping to be strong. This is valid in the most experiments on quasi-2D organic metals where the amplitude of the second harmonic does not usually exceed $5 \%$ of the first harmonic.

So, in spite of the approximations made in the above analysis, the proposed theoretical description is valid in a large domain of parameters which one has in real experiments on quasi-2D organic metals. For example, above result concerning the slow oscillations and the phase of beats of the $\mathrm{SdH}$ oscillations is important for an experimental study of these effects in $\beta$-(BEDT-TTF $)_{2} \mathrm{IBr}_{2}$ (see, e.g. [20], [21]). The proposed results may be also used for heterostructures with large enough interlayer jumping.

The author thanks M.V. Kartsovnik, W. Biberacher, A.M. Dyugaev and I. Vagner for encouragement and stimulating discussions. The work was supported by the EU ICN contract HPRI-CT-1999-40013 and RFBR No. 00-02-17729a.

\section{APPENDIX A: TRANSFORMATION OF THE SUMS OVER LLS TO THE SUMS OVER HARMONICS}

To transform the sums over LL number into the harmonic sums we shall apply the Poisson summation formula [23]

$$
\sum_{n=n_{0}}^{\infty} f(n)=\sum_{k=-\infty}^{\infty} \int_{a}^{\infty} e^{2 \pi i k n} f(n) d n
$$

where $a \in\left(n_{0}-1 ; n_{0}\right)$. This formula is valid for arbitrary function $f(n)$. The electron velocity is determined from the dispersion relation (11) as

$$
\begin{aligned}
v_{z}(\epsilon, n) & \equiv \frac{\partial \epsilon\left(n, k_{z}\right)}{\hbar \partial k_{z}}=-\frac{2 t d}{\hbar} \sin \left(k_{z} d\right)= \\
& =\frac{d}{\hbar} \sqrt{4 t^{2}-\left(\epsilon-\hbar \omega_{c}(n+1 / 2)\right)^{2}}
\end{aligned}
$$

The sum in (5) now becomes

$$
\begin{aligned}
\sum_{n}\left|v_{z}(\epsilon, n)\right| & =\sum_{n=0}^{\infty} \frac{d}{\hbar} \sqrt{4 t^{2}-\left(\epsilon-\hbar \omega_{c}\left(n+\frac{1}{2}\right)\right)^{2}}= \\
& =\frac{d}{\hbar} \hbar \omega_{c} \sum_{k=-\infty}^{\infty} \int_{0}^{\infty} d n e^{2 \pi i k\left(n-\frac{1}{2}\right)} \sqrt{\left(\frac{2 t}{\hbar \omega_{c}}\right)^{2}-\left(\frac{\epsilon}{\hbar \omega_{c}}-n\right)^{2}}
\end{aligned}
$$




$$
\begin{aligned}
& =\frac{d}{\hbar} \hbar \omega_{c} \sum_{k=-\infty}^{\infty}(-1)^{k} \exp \left(\frac{2 \pi i k \epsilon}{\hbar \omega_{c}}\right) \int_{-\infty}^{\infty} d x e^{2 \pi i k x} \sqrt{\left(\frac{2 t}{\hbar \omega_{c}}\right)^{2}-x^{2}} \\
& =\sum_{k=-\infty}^{\infty} \frac{d t}{\hbar} \frac{(-1)^{k}}{k} \exp \left(\frac{2 \pi i k \epsilon}{\hbar \omega_{c}}\right) J_{1}\left(\frac{4 \pi k t}{\hbar \omega_{c}}\right)
\end{aligned}
$$

In this formula for the zeroth harmonic $k=0$ one should use the expansion $J_{1}(k x) / k=x / 2$.

\section{APPENDIX B: MAGNETIZATION}

The first harmonic of the oscillating part of magnetization is given by (see [6], formula 6)

$$
\tilde{M}(B)=\frac{2 N_{L L} \varepsilon_{F}}{\pi B} \sin \left(\frac{2 \pi\left(\varepsilon_{F}+\tilde{\mu}(B)\right)}{\hbar \omega_{c}}\right) J_{0}\left(\frac{4 \pi t}{\hbar \omega_{c}}\right) R_{T} R_{S} R_{D}\left(\varepsilon_{F}\right) .
$$

where the oscillating part of the chemical potential is ( [6], formula 5)

$$
\tilde{\mu}(B)=\frac{\hbar \omega_{c}}{\pi\left(1+n_{R}\left(\varepsilon_{F}\right)\right)} \times \sin \left(\frac{2 \pi\left(\varepsilon_{F}+\tilde{\mu}(B)\right)}{\hbar \omega_{c}}\right) J_{0}\left(\frac{4 \pi t}{\hbar \omega_{c}}\right) R_{T} R_{S} R_{D}
$$

The entanglement of magnetization oscillations with the oscillations of the Dingle factor (9) produces an additional term

$$
\begin{gathered}
\propto \sin \left(\frac{2 \pi \varepsilon_{F}}{\hbar \omega_{c}}\right) J_{0}\left(\frac{4 \pi t}{\hbar \omega_{c}}\right) \times \cos \left(\frac{2 \pi \varepsilon_{F}}{\hbar \omega_{c}}\right) J_{0}\left(\frac{4 \pi t}{\hbar \omega_{c}}\right) \\
=\frac{1}{2} \sin \left(\frac{4 \pi \varepsilon_{F}}{\hbar \omega_{c}}\right) J_{0}^{2}\left(\frac{4 \pi t}{\hbar \omega_{c}}\right)
\end{gathered}
$$

which give rise to the second harmonic but makes zero contribution to the slow oscillations of magnetization.

The entanglement with the oscillations of the chemical potential (B2) produces the term

$$
\begin{gathered}
\propto \sin \left(\frac{2 \pi\left(\varepsilon_{F}+\tilde{\mu}(B)\right)}{\hbar \omega_{c}}\right)-\sin \left(\frac{2 \pi \varepsilon_{F}}{\hbar \omega_{c}}\right) \\
=\sin \left(\frac{2 \pi \varepsilon_{F}}{\hbar \omega_{c}}\right)\left[\cos \left(\frac{2 \pi \tilde{\mu}(B)}{\hbar \omega_{c}}\right)-1\right]+\cos \left(\frac{2 \pi \varepsilon_{F}}{\hbar \omega_{c}}\right) \sin \left(\frac{2 \pi \varepsilon_{F}}{\hbar \omega_{c}}\right) J_{0}\left(\frac{4 \pi t}{\hbar \omega_{c}}\right) \frac{2 R_{T} R_{D}}{1+n_{R}}
\end{gathered}
$$

which also contribute only to the second harmonics (or higher harmonics) but not to slow oscillations.

[1] Shoenberg D. "Magnetic oscillations in metals", Cambridge University Press 1984

[2] T. Ishiguro, K. Yamaji and G. Saito, Organic Superconductors, 2nd Edition, Springer-Verlag, Berlin, 1998.

[3] J. Wosnitza, Fermi Surfaces of Low-Dimensional Organic Metals and Superconductors (Springer-Verlag, Berlin, 1996); M.V. Kartsovnik and V.N. Laukhin, J. Phys. I France 6, 1753 (1996); J. Singleton, Rep. Prog. Phys. 63, 1111 (2000).

[4] V.M. Gvozdikov, Fiz. Tverd. Tela 26, p. 2574 (1984) [Sov. Phys. Solid State 26 (9), p. 1560 (1984)]; K. Jauregui, V.I. Marchenko, I.D. Vagner, Phys. Rev. B 41, 12922 (1990); M.A. Itskovsky, T. Maniv and I.D. Vagner, Phys. Rev. B 61, 14616 (2000); P. Grigoriev and I. Vagner, Pis'ma Zh. Eksp. Teor. Fiz., 69, 139 (1999) [JETP Letters 69, 156 (1999)]; T. Champel and V.P. Mineev, Phil. Magazine B 81, 55 (2001); T. Champel, Phys. Rev. B 64, 54407 (2001).

[5] N. Harrison et al., Phys. Rev. B 54, 9977 (1996).

[6] P. Grigoriev, Zh. Eksp. Teor. Fiz. 119(6), 1257 (2001) [JETP 92, 1090 (2001)].

[7] A.E.Datars and J.E.Sipe, Phys. Rev. B 51, 4312 (1995) 
[8] P. Moses and R.H. McKenzie, Phys. Rev. B 60, 7998 (1999)

[9] V.M. Gvozdikov, Fiz. Nizk. Temp. 27 (9/10), p. 956 (2001) [Sov. J. Low Temp. Phys. 27(9/10) (2001)].

[10] N.S. Averkiev, L.E. Golub, S.A. Tarasenko and M. Willander, J. of Phys. Cond. Matter 13, 2517 (2001).

[11] A.P. Mackenzie, S.R. Julian, A.J. Diver et al., Phys. Rev. Lett. 76, 3786 (1996); C. Bergemann, S.R. Julian, A.P. Mackenzie, S. NishiZaki and Y. Maeno, Phys. Rev. Lett. 84, 2662 (2000).

[12] J. Wosnitza, G.W. Crabtree, K.D. Carlson, H.H. Wang and J.M. Williams, Physica B 194-196, 2007 (1994); J. Wosnitza, G. Goll, D. Beckmann, S. Wanka, D. Schweitzer and W. Strunz, J. Phys. I France 6, 1597 (1996).

[13] A.A. Abrikosov, Fundamentals of the theory of metals, North-Holland, 1988

[14] H. Weiss, M. V. Kartsovnik, W. Biberacher, E. Balthes, A.G.M. Jansen, and N.D. Kushch, Phys. Rev. B 60, R16259 (1999).

[15] M.Schiller, W.Schmidt, E.Balthes et al., Europhys. Lett. 51, 82 (2000).

[16] M. Kartsovnik, V. Laukhin, V. Nizhankovskii and A. Ignat'ev, Pis'ma Zh. Eksp. Teor. Fiz. 47, 302 (1988) [Sov. Phys. JETP Lett. 47, 363 (1988)]; M. Kartsovnik, P. Kononovich, V. Laukhin and I. Schegolev, Pis'ma Zh. Eksp. Teor. Fiz. 48, 498 (1988) [Sov. Phys. JETP Lett. 48, 541 (1988)]; M. Kartsovnik, P. Kononovich, V. Laukhin, S. Pesotskii and I.F.Schegolev, Pis'ma Zh. Eksp. Teor. Fiz. 49, 453 (1989) [Sov. Phys. JETP Lett. 49, 519 (1989)]; M. Kartsovnik, V. Laukhin and S. Pesotskii, Fiz. Nizk. Temp. 18, 22 (1992) [Sov. J. Low Temp. Phys. 1813 (1992)].

[17] T. Terashima, S. Uji, H. Aoki, M. Tamura, M. Kinoshita and M. Tokumoto, Solid State Commun. 91, 595 (1994); E. Ohmichi, H. Ito, T. Ishiguro, G. Saito and T. Komatsu, Phys. Rev. B 57, 7481 (1998).

[18] B. Narymbetov, N. Kushch, L. Zorina et al., Eur. Phys. J. B 5, 179 (1998); T. Togonidze, M. Kartsovnik, J. Perenboom, N. Kushch and H. Kobayashi, Physica B 294-295 435 (2001).

[19] L. Balicas, J. Brooks, K. Storr, D. Graf, S. Uji, H. Shinagawa, E. Ojima, H. Fujiwara, H. Kobayashi, A. Kobayashi and M. Tokumoto, cond-mat/0008287 (2000).

[20] P.D. Grigoriev, M.V. Kartsovnik, W. Biberacher, N.D. Kushch, P. Wyder, Phys. Rev. B 65, 60403(R) (2002)

21] M.V. Kartsovnik, P.D. Grigoriev, W. Biberacher, N.D. Kushch, P. Wyder, submitted to Phys. Rev. Lett. condmat/0203279) (2002)

[22] G. Mahan "Many-Particle Physics", 2nd ed., Plenum Press, New York, 1990, (§7.1)

[23] "Standard Mathematical Tables and Formulae", CRC Press, (1996)

[24] In real layered compounds the impurity distribution may have periodic modulation in the direction perpendicular to the layers and with period equal to the interlayer distance. However, in Born approximation this leads only to a constant factor in (11) which renormalizes the Dingle temperature.

[25] This fact has evident physical meaning. The matrix element of scattering by a point-like impurity $U$ is the same for all transitions not changing electron energy. Hence, the scattering rate $1 / \tau(\epsilon)=-2 \operatorname{Im} \Sigma^{R} / \hbar$ is proportional to the number of states to which an electron may scatter, that is, to the density of states at a given energy.

[26] P.D. Grigoriev, M.V. Kartsovnik, W. Biberacher and P. Wyder, cond-mat/0108352 (2001) (unpublished).

[27] This fact can be understood in a different way: slow oscillations originate from local electron motion and are not sensitive to global variations of the position of the chemical potential with respect to the bottom of the conducting band.

[28] Yamaji K., J. Phys. Soc. Jpn. 58, 1520 (1989).

[29] Yasunari Kurihara, J. Phys. Soc. Jpn. 61, 975 (1992). 